\title{
Towards a silicon laser based on emissive structural defects
}

\author{
Anatolii V. Yukhnevich * \\ Research Institute for Physical and Chemical Problems of the Belarussian State University, 14 Leningradskaya Str., Minsk 220030, Belarus
}

Received 8 August 2006; received in revised form 4 January 2007; accepted 25 January 2007

The review of this paper was arranged by Prof. Y. Arakawa

\begin{abstract}
The possibility of creating silicon lasers based on specific few-atomic structural imperfections of a silicon crystal is discussed. These imperfections differ substantially from other defects of the crystal in their high photon emissivity, which is a consequence of zero-phonon optical transitions between the localized electron states of the defects considered. A simple model of basic electron processes in the active region of the laser crystal is proposed. Such lasers can operate in the quantum energy range from 0.4 to $1.1 \mathrm{eV}$ at temperatures less than $100 \mathrm{~K}$ in a continuous mode. An injection way of excitation and current modulation of the emission intensity up to gigahertz frequencies are possible. The main difficulty in manufacturing such devices is the nonselectivity of today's radiation methods of forming emissive centers in silicon crystals. This difficulty may be obviated by using promising nanotechnology methods of fabrication of the lasers considered.
\end{abstract}

(C) 2007 Elsevier Ltd. All rights reserved.

Keywords: Silicon laser; Silicon optoelectronics; Silicon microphotonics

\section{Introduction}

Monocrystalline silicon (mc-Si) is the basic material of the contemporary electronics and, according to many forecasts, in the future it will remain a major material of information engineering (see also [1]). One of the promising ways for upgrading the silicon devices is assimilation of optical (photon) representation of signals. For this purpose, different silicon photon devices have been developed successfully. However, efficient photon emitters are lacking in a complex of such devices. As is known, this is caused by 'indirect' band-gap of $\mathrm{mc}-\mathrm{Si}$, which leads to a low probability of band-to-band optical electronic transitions and, accordingly, of creating a laser based on these transitions. The expectation of obvious positive effects from the development of silicon microphotonics increases the activity in the search for the ways of manufacturing silicon lasers

\footnotetext{
* Tel.: +375 17 2224510; fax: +375 172264696 .

E-mail address: yukhnevich@bsu.by
}

and radiation amplifiers. Among these approaches is the most commonly encountered use of the specific properties of low-size silicon crystals, impurity rare-earth ions, and the Raman effect [2-4]. Up to now, the luminescence efficiency achieved in these experiments has been inadequate for realizing lasing in the preferable current-injection continuous mode.

In the present paper, we consider (and offer for development) the promising, in our opinion, but as yet insufficiently studied approach to the creation of silicon lasers. It is based on the use of the luminescence of specific structural imperfections of silicon crystals which can be called 'emissive structural defects' (ESDs). No mention has been made of this approach in reviews [2,3] devoted to the problem.

\section{Emissive structural defects}

ESDs differ from the multitude of other defects in mc-Si by the high probability of photon emission when free 
electrons, holes, and excitons recombine on them at low temperature. For example, at $T<150 \mathrm{~K}$ this probability is at least $10^{3}$ times higher than that for band-to-band emission and for emissive recombination of free particles on well-known 'shallow' donors and acceptors. The first ESD in mc-Si was revealed among radiation damages through the mediation of an intensive narrow emission line with an energy of $970 \mathrm{meV}$ in the low-temperature electroluminescent spectra of Si-diodes irradiated by ${ }^{60} \mathrm{Co}$ gamma-rays [5]. Further, carrying out the study of electro-, cathodo-, and photo-luminescence of different mc-Si samples, we have revealed a number of new ESDs via analogous characteristic narrow lines (with spectral linewidth of about $0.1 \mathrm{meV}$ ) in their individual emission spectra. It was established that conditions for the formation of each such defect are various and depend on the characteristic features of the initial crystal, a type of irradiation (gamma-rays, fast electrons, neutrons, ions), temperature and time of annealing of irradiated and nonirradiated samples. It was shown that the narrow spectral lines of luminescence owe their origin to zero-phonon emissive electron transitions (an optical analogue of the Mossbauer

Table 1

Emissive structural defects (ESD) in silicon monocrystals

\begin{tabular}{llllll}
\hline ESD $^{\mathrm{a}}$ & $\gamma, e$ & $n$ & $i$ & $t$ & $\Delta t,{ }^{\circ} \mathrm{C}$ \\
\hline 1062 & - & + & + & & $300-550$ \\
1040 & - & + & + & & $250-450$ \\
1018 & - & + & + & & $<500$ \\
$1010^{\mathrm{b}}$ & - & - & $\mathrm{Ar}$ & & $<500$ \\
1004 & + & + & + & & $300-500$ \\
997 & + & + & + & & $150-350$ \\
995 & + & + & + & + & $500-550$ \\
985 & - & + & + & & $<350$ \\
970 & + & + & + & & $<300$ \\
967 & - & + & + & & $<350$ \\
962 & - & + & + & & $<250$ \\
957 & + & + & + & & $<200$ \\
953 & + & + & + & & $<350$ \\
945 & + & + & + & + & $500-550$ \\
943 & - & + & + & & $<450$ \\
926 & + & + & + & + & $350-550$ \\
924 & + & + & + & & $100-250$ \\
922 & + & + & + & + & $350-550$ \\
920 & + & + & + & & $350-450$ \\
854 & + & + & + & & $350-450$ \\
790 & + & + & + & & $<450$ \\
767 & + & + & + & + & $350-550$ \\
488 & + & + & + & & $<200$ \\
\hline
\end{tabular}

${ }^{a}$ Each ESD is referred to as the energy (in $\mathrm{meV}$ ) of the most intensive no-phonon line in its own emission spectrum [6]. The symbols $\gamma, e, n$, and $i$ correspond to the Si-crystal irradiation by gamma-rays of ${ }^{60} \mathrm{Co}$, electrons with an energy less than $10 \mathrm{MeV}$, fast neutrons and ions, respectively. The signs in the columns mentioned relate to the cases when the ESD was observed $(+)$ or not observed $(-)$. The column ' $t$ ' presents the ESDs observed $(+)$ in the nonirradiated crystals annealed in the specified temperature interval ('the emissive thermodefects'); the other ESDs were not observed in the crystals mentioned. The value $\Delta t$ is the anneal temperature interval in which defect was formed after (or without) the crystal irradiation.

${ }^{\mathrm{b}}$ This ESD was formed after irradiation by Ar-ions only. effect) between the localized electron states of different ESDs, which are few-atomic ('point') defects with a strictly distinct atomic architecture. The quantum energy corresponding to the most intensive narrow line in their individual spectra is strictly specific to each defect and does not depend on the conditions of its formation. The numerical value of this energy is a reliable attribute for optical identification of each ESD, and this value can serve as its individual name. The revealed emissive defects named precisely in this way are presented in Table 1, where the main conditions of their formation are given as well. The possibility of creating silicon lasers based on these defects was mentioned previously (see review of our works [6]). These and other ESDs were also observed and studied by other research groups (see, for example, review [7]). The main objective of the present paper is to estimate theoretically for the first time, in more detail, the possibility of creating silicon lasers based on ESDs using our and other experimental data.

\section{Model of lasing}

The diagram of the basic electronic processes in the active region of the devices considered is shown in Fig. 1. The diagram reflects the following assumptions to be made in the proposed simple model of the processes. The silicon monocrystal (mc-Si in the diagram) contains only one type of laser centers (ESD) with concentration $N$. The electronic system of each of such centers is characterized by two nondegenerated electronic localized states with energy levels $E_{1}$ and $E_{2}$. An optical activity of the ESDs is determined by spontaneous and induced zero-phonon radiative transitions between these levels. The ESDs are excited by the energy of recombination of free nonequilibrium electronhole pairs and/or excitons (with concentration $n$ ) at the centers (process $W$ ). The generation of nonequilibrium electrons and holes (process $G$ ) is initiated by any method which will transfer the energy $E_{G}>\left(E_{C}-E_{V}\right)$ to an electronic system of the crystal. The efficiency of the excitation of the laser centers is reduced predominantly due to the process $Q$ which is nonradiative (thermal) recombination of nonequilibrium electron-hole pairs and excitons on other structural defects characterized by an effective con-

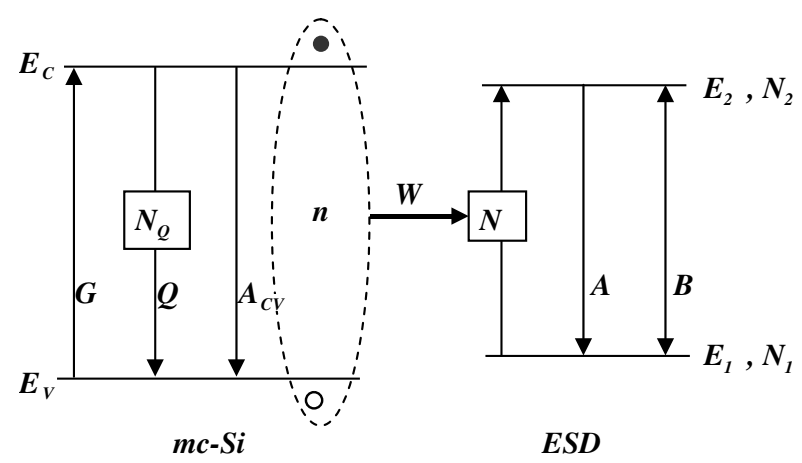

Fig. 1. Diagram of the basic electronic processes in active region of the lasers considered. 
centration $N_{Q}$ and recombination factor $Q$. The low-intensity 'intrinsic' band-to-band radiative recombination (process $\left.A_{\mathrm{CV}}\right)$ and nonradiative deactivation of the excited states of the laser centers are neglected. The relations between the parameters of the model are determined by the following set of coupled rate equations:

$$
\begin{aligned}
\mathrm{d} n / \mathrm{d} t= & G-W \cdot N_{1} \cdot n-Q \cdot N_{Q} \cdot n, \\
\mathrm{~d} N_{1} / \mathrm{d} t= & -W \cdot n \cdot N_{1}+A \cdot N_{2} \\
& +B_{21} \cdot N_{2} \cdot \rho(v)-B_{12} \cdot N_{1} \cdot \rho(v), \\
\mathrm{d} N_{2} / \mathrm{d} t= & +W \cdot n \cdot N_{1}-A \cdot N_{2}-B_{21} \cdot N_{2} \cdot \rho(v) \\
& +B_{12} \cdot N_{1} \cdot \rho(v),
\end{aligned}
$$

where $N_{1}$ and $N_{2}$ are the concentrations of the emissive centers in the ground and excited states, respectively, with $N_{1}+N_{2}=N ; G$ is the volume density of the crystal excitation; $W$ and $A$ are the factor of excitation and the probability of spontaneous emission of the laser center; $B_{21}$ and $B_{12}$ are the probabilities of induced emission and absorption of quanta with energy $h v=\left(E_{2}-E_{1}\right)$ in an emission field with the energy density $\rho(v)$ (here, $B_{21}=B_{12}=B$ are the Einstein coefficients). Within the framework of the given model, in accordance with Eq. (1), the transparency threshold of the laser active region (at zero inverse population of laser levels, i.e., at $N_{2}=N_{1}$ ) is achieved at the following density of stationary excitation:

${ }^{0} G=A \cdot N / 2+A \cdot Q \cdot N_{Q} / W$

without taking into account the optical loss and considering that $B_{21} \cdot N_{2} \cdot \rho(v)=B_{12} \cdot N_{1} \cdot \rho(v)$ at the threshold.

\section{4. 'Perfect' laser}

In order to be oriented in the numerical values of the operating conditions of the devices considered, let us estimate the threshold excitation density ${ }^{0} G_{\mathrm{P}}$ of the 'perfect' laser based on the zero-phonon transitions of the ' 970 meV' ESD. The latter can be characterized by the following own parameters: $A=5 \times 10^{8} \mathrm{~s}^{-1}, W \approx Q \approx$ $10^{-8} \mathrm{~cm}^{3} \mathrm{~s}^{-1}$ at $T<20 \mathrm{~K}$ in Si-samples irradiated by a low-exposure dose of gamma-rays or fast electrons. (These numerical estimations are the generalized result of the agreement between the experimental data of the works cited in [6] and solutions of Eq. (1)). Let us define the "perfection' of the laser by the absence of optical and thermal losses $\left(N_{Q}=0\right)$. Then, in accordance with $(2),{ }^{0} G_{\mathrm{P}}=$ $A \cdot N / 2$. As an example, we shall consider a device with an active region in the form of a layer of thickness $d=1 \mu \mathrm{m}$ with $N\left(\right.$ (' $\left.970 \mathrm{meV}^{\prime}\right)=10^{15} \mathrm{~cm}^{-3}$; energy $E_{\mathrm{G}}=$ $1.2 \mathrm{eV}$ is needed for each act of crystal excitation. Then the surface threshold density of excitation of the active layer is equal to ${ }^{0} G_{\mathrm{SP}}={ }^{0} G_{\mathrm{P}} \cdot d \cdot E_{G}=0.5 \cdot A \cdot N \cdot d \cdot E_{G} \approx$ $5 \mathrm{~W} \mathrm{~cm}^{-2}$. This excitation density of the 'perfect' active $i$-layer in the $p^{+}-i-n^{+}$diode laser will be achieved at the threshold density of injection current ${ }^{0} J_{\mathrm{P}} \approx 4 \mathrm{~A} \cdot \mathrm{cm}^{-2}$, if this whole current is the current of radiative recombination of electrons and holes in the $i$-layer. The internal efficiency of such a device is $\left(E_{2}-E_{1}\right) / E_{G} \approx 0.8$.

These comparatively low threshold pump power and injection current of 'perfect' lasers indicate that a continuous operation mode of actual lasers of the type studied is realizable. These threshold conditions are comparable with those present in the best contemporary and promising devices based on semiconductors with a 'direct' band-gap operating at low temperatures [8]. According to solutions of Eq. (1) for the ' $970 \mathrm{meV}$ ' center, the rate of the basic electronic processes shows the possibility of obtaining gigahertz modulation frequencies of emission intensity on electrical or optical pumping of the devices based on this ESD. The estimates of the parameters of other ESDs in Table 1 allow us to consider most of them as possible laser centers as well. In this regard, apart from ' $970 \mathrm{meV}$ ', the most interesting objects are the following ESDs: ' $1018 \mathrm{meV}$ ', ' $790 \mathrm{meV}$ ', and ' $488 \mathrm{meV}$ '. In addition to the main no-phonon line, some of ESDs have one or more narrow lines (different in nature (see [6,7])) with lower intensity in their own emissive spectra, and some of these lines can be used as the laser line as well. It should be noted that the probability of zero-phonon emissive electron transitions (parameter $A$ in the model) decreases with increasing temperature, following the temperature dependences which are typical of the optical analogue of the Mossbauer effect [9]. As a result, low operating temperatures $(<100 \mathrm{~K})$ in the devices discussed are required.

\section{Difficulties and prospects of realization}

The basic difficulty in creating the lasers considered is the nonselectivity of present methods of ESD formation in mc-Si. The majority of the throughly investigated ESDs are radiation defects. They are formed during irradiation of a crystal by high-energy particles simultaneously with a great quantity of nonemissive (thermal) recombination centers. The total concentration of the latter is much higher than the ESD concentration for all kinds of irradiation. Within the framework of the model examined, this means that $N_{Q} / N \gg 1$. With this relationship between the concentrations of the 'useful' and 'harmful' centers in the device, the greater part of the excitation energy will be spent on crystal heating. This complicates the development of continuous mode lasers if their active region is formed by radiation methods. This is especially true in regard to the lasers intended to be exploited at 'high' temperatures $(>80 \mathrm{~K})$. However, the pulse operating mode in such devices is possible. It is pertinent to note that evidences of stimulated emission from mc-Si at low temperatures $(<80 \mathrm{~K})$ with the wavelength corresponding to zero-phonon line of ' $970 \mathrm{meV}$ ' centers formed in the course of irradiation of the crystals by ions were first observed in specific Si-structures on photon band-to-band excitation [10].

Most likely, promising technologies of active region formation will be based not only on radiation and thermal reorganization of the perfect crystal lattice, but also on 
the processes of a subtle synthesis of the crystal. Such processes (like local epitaxy) must provide the way for local directed formation of the atomic structure of a growing crystal. Rapid progress in nanotechnology (atomic engineering) gives hope for a fast development of new physico-chemical approaches to a low-temperature synthesis of thin mc-Si layers containing only one predetermined type of 'useful' structure imperfections, for example, only the ' $970 \mathrm{meV}$ ' centers, with a minimum of 'harmful' recombination and scattering centers. It should be noted that the problem of directed formation of the discussed few-atomic ESDs in the active region of the lasers considered is in many respects similar to a problem of formation of quantum dots - small-sized multiatomic photon emitters in the active region of the most effective modern lasers based on semiconductors with a 'direct' forbidden zone [8]. It is possible to consider the ESDs as 'quantum dots' of extremely small sizes with the greatest possible identity (the smallest dispersion) of parameters, which is a desirable but as yet inaccessible property of sets of quantum dots in the best present-day semiconductor lasers [11].

\section{Conclusions}

The paper has presented the arguments in favour of the fact that the own properties of the known emissive structural defects (ESDs) in silicon monocrystal allow one to create, on their basis, promising silicon lasers and light amplifiers operating in the range of wavelengths from 1 to $3 \mu \mathrm{m}$. Such devices will be able to work at temperatures below $100 \mathrm{~K}$ in a continuous mode on current injection and with other methods of excitation. A simple model of the basic electronic processes in the active region of the laser crystal is proposed. The model makes it possible to estimate the threshold conditions of the discussed devices from the basic individual parameters of the ESD used. It is hoped that the devices considered will take a worthy place in the future silicon low-temperature optoelectronics.

\section{References}

[1] Stormer HL. Silicon forever! Really? Solid-State Electron 2006;50:516-9.

[2] Pavesi L, Gaponenko S, Dal Negro L, editors. Towards the first silicon laser. Dordrecht: Kluwer Academic Publishers; 2003. p. 482.

[3] Pavesi L. Routes toward silicon-based lasers. Mater Today 2005; $8: 18-25$.

[4] Rong H, Jones R, Liu A, Cohen O, Hak D, Fang A, et al. A continuous-wave Raman silicon laser. Nature 2005;433:725-8.

[5] Yukhnevich AV. The structure of the spectrum of the radiative capture of holes by A-centers in silicon. Soviet Physics-Solid State 1965;7:259-60.

[6] Yukhnevich AV. Some features of an atomic structure of silicon monocrystals. Selected research works of the Belarusian State University, vol. 5. Minsk: BSU; 2001. p. 89-122 [in Russian].

[7] Davies G. The optical properties of luminescence centres in silicon. Phys Reps 1989;176:83-188.

[8] Alferov ZI. The double heterostructure concept and its applications in physics, electronics, and technology. Rev Mod Phys 2001; 73:767-82.

[9] Maradudin AA. Theoretical and experimental aspects of the effects of point defects and disorder on the vibrations of crystals. Solid State Phys 1966;18:273-420.

[10] Cloutier SG, Kossyrev PA, Xu J. Optical gain and stimulated emission in periodic nanopatterned crystalline silicon. Nature Mater 2005;4:887-91.

[11] Asryan LV, Suris RA. Theory of threshold characteristics of quantum dot lasers: Effect of quantum dot parameter dispersion. International J. High Speed Electron. Syst., Special Issue on 'Quantum Dot Heterostructures - Fabrication, Application, Theory' 2002;12:111-76. 